\title{
The distance to an outer Galaxy star forming region
}

\author{
K. Hachisuka ${ }^{1}{ }^{2}$, A. Brunthaler ${ }^{2}$, M. J. Reid ${ }^{3}$ and K. M. Menten ${ }^{2}$ \\ ${ }^{1}$ Shanghai Astronomical Observatory, 80 Nandan Road, Shanghai, 200030, China \\ email: khachi@shao.ac.cn \\ ${ }^{2}$ Max-Planck-Institut für Radioastronomie, Auf dem Hügel 69, Bonn, Germany \\ ${ }^{3}$ Harvard-Smithsonian Center for Astrophysics, 60 Garden Street, Cambridge, MA, USA
}

\begin{abstract}
We performed phase-referencing VLBI astrometric observations of the $\mathrm{H}_{2} \mathrm{O}$ maser source IRAS $02395+6244$, located well beyond the solar circle. We measured its heliocentric distance to be $5.49 \pm 0.80 \mathrm{kpc}$, implying a Galactocentric distance of $12.5 \pm 0.5 \mathrm{kpc}$ and a distance of $270 \pm 40 \mathrm{pc}$ above the Galactic plane.
\end{abstract}

Keywords. Galaxy: structure, masers, astrometry

\section{Introduction}

In the past, the structure and size-scale of our Galaxy have been mainly determined by translating measured radial velocities into distances with the help of a kinematic model - "the rotation curve". (e.g. Nakanishi \& Sofue 2003). Such methods originally proved the existence of the Milky Way's spiral structure and are still in use today. However, the resulting "kinematic distances" strongly depend on the Galactic rotation model chosen and peculiar motions may render them questionable (see, e.g., Xu et al. 2006). This is particularly true for the outer galaxy, since the Galactic rotation speed there has still a larger uncertainty than in the inner Galaxy (Brand \& Blitz 1993). Consequently, many uncertainties remain on the structure and distance scale of the outer Galaxy.

Recently, distances of Galactic maser sources have been determined by annual parallax measurement using the phase-referencing VLBI technique (e.g. Hachisuka et al. 2006 and see contribution by M. Reid in these proceedings). This method can measure the annual parallax of Galactic objects out to many kiloparsecs with errors of a few percent at a kpc (scaling with distance), allowing direct distance measurements for objects located near the edge of the stellar disk of our Galaxy.

Wouterloot et al. (1993) searched for $\mathrm{H}_{2} \mathrm{O}$ maser sources in the outer galaxy associated with ${ }^{12} \mathrm{CO}$ emission and found a number of them, for which their kinematic distances indicated large distances from the Galactic center. We selected one of these, associated with IRAS $02395+6244$, as the target source for an annual parallax measurement.

\section{Observations}

We used the VLBA to observe the IRAS $02395+6244 \mathrm{H}_{2} \mathrm{O}$ maser source using phasereferencing VLBI techniques. The observations were performed at 5 epochs spread over a year. For position reference we used an ICRF source separated by $0.5 \mathrm{deg}$ from the target source. See $\mathrm{Xu}$ et al. (2006) for a description of the data reduction procedures. 


\section{Results}

Several $\mathrm{H}_{2} \mathrm{O}$ maser components could be imaged after the calibration. We determined their positions and traced individual maser components between different epochs carefully, monitoring their intrinsic variations. We found an $\mathrm{H}_{2} \mathrm{O}$ maser component that was stable over the course of the observations and determined its annual parallax.

Figure 1 shows the change in position versus time of that $\mathrm{H}_{2} \mathrm{O}$ maser component. We estimate the annual parallax to be $0.182 \pm 0.026$ mas, which corresponds to a heliocentric distance of $5.49 \pm 0.80 \mathrm{kpc}$, or $12.5 \pm 0.9 \mathrm{kpc}$ from the Galactic center for $R_{0}=8.0 \mathrm{kpc}$ (Reid 1993). The Galactic latitude of the source is $2.8 \mathrm{deg}$, hence it is located $270 \pm 40$ pc above the Galactic plane.
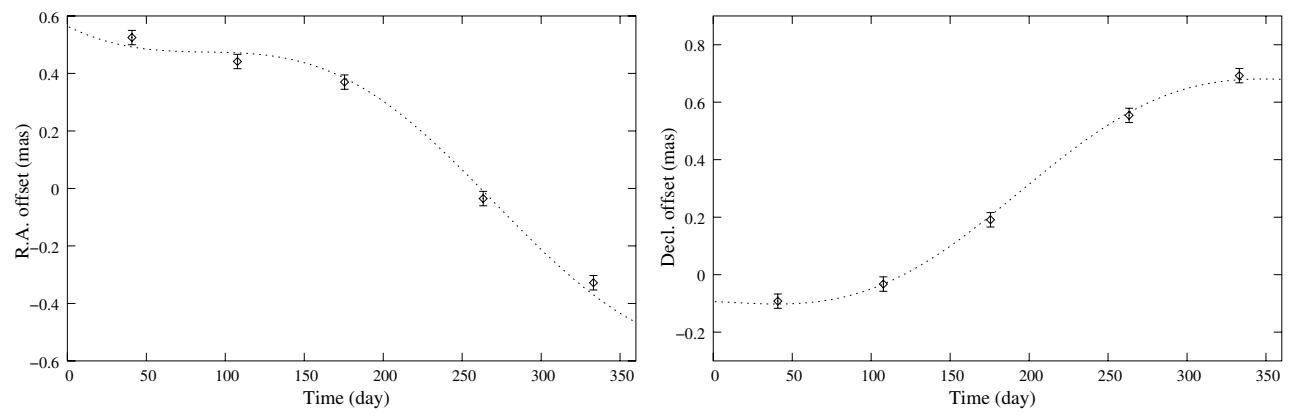

Figure 1. Change of the position with time for an $\mathrm{H}_{2} \mathrm{O}$ maser component associated with IRAS $02395+6244$ with respect to a background extragalactic source. Displacements in right ascension (left panel) and declination (right panel) are shown. Dotted lines show the best-fit result for a proper motion and an annual parallax.

The kinematic distance of our $\mathrm{H}_{2} \mathrm{O}$ maser source with an LSR velocity of $-72 \mathrm{~km} \mathrm{~s}^{-1}$ is $8.7 \mathrm{kpc}$ from the Sun, assuming a flat rotation curve and the IAU recommended values of $\Theta_{0}=220 \mathrm{~km} \mathrm{~s}^{-1}$ and $R_{0}=8.5 \mathrm{kpc}$. The kinematic distance is significantly larger than the parallactic distance of $5.49 \pm 0.80 \mathrm{kpc}$. Such discrepancies between kinematic and annual parallaxes have been found for other Galactic maser sources in the outer Galaxy (Hachisuka et al. 2006, Xu et al 2006; see contribution by M. Reid). Also, after removing the effects of Galactic rotation, we find a very large peculiar motion at $\approx 30 \mathrm{~km} \mathrm{~s}$. More observations like these should lead to better a understanding of the size, dynamics, and dark matter halo of the Galaxy.

\section{Acknowledgements}

This work has been partially supported by the National Natural Science Foundation of China (grants 10573029 and 10625314) and the Knowledge Innovation Program of the Chinese Academy of Sciences (Grant No. KJCX2-YW-T03), and the National Key Basic Research Development Program of China (No. 2007CB815405).

\section{References}

Brand, J. \& Blitz, L., 1993, A\&A, 275, 67

Hachisuka, K., Brunthaler, A., Menten, K. M., Reid, M. J. et al., 2006, ApJ, 645, 337

Nakanishi, H. \& Sofue, Y., 2003, PASJ, 55, 191

Reid, M. J., 1993, ARAA, 31, 345

Wouterloot, J. G. A., Brand, J., \& Fiegle, K., 1993, A\&\&AS, 98, 589

Xu, Y., Reid, M. J., Zheng, X. W., \& Menten, K. M., 2006, Science, 311, 54 